\title{
Diagnostic Paracentesis in the Acute Abdomen
}

\author{
W. N. W. BAKER,* M.B., B.S., F.R.C.S. ; D. B. MACKIE,* M.B., M.CHIR., F.R.C.S. \\ J. F. NEWCOMBE, $†$ M.CHIR., F.R.C.S.
}

Bnix. med.F., 1967, 3, 146-149

Diagnostic paracentesis of the peritoneal cavity in abdominal emergencies has been practised in the United States for many years, but has never become popular in this country (Morris, 1966).

The first comprehensive study of the technique was carried out by Neuhof and Cohen (1926), who reported enthusiastically on its use as a diagnostic aid in the evaluation of closed abdominal injuries, acute pancreatitis, and primary pneumococcal or streptococcal peritonitis. Though no cases were reported in their paper, they also predicted a wider use for the test in the diagnosis of ruptured hollow viscera and bleeding ectopic pregnancies.

Later studies have confirmed these observations, especially in relation to blunt abdominal trauma (Thompson and Brown, 1954 ; Economy et al., 1960). Williams and Zollinger (1959) reviewed 273 abdominal injuries, $80 \%$ of which were nonpenetrating, and reported that needle paracentesis had proved accurate in $79 \%$ of these cases. They compared the relative diagnostic values of paracentesis and $x$-ray findings in the same series, and found that radiology was more valuable in injuries to kidney and bladder (where paracentesis is usually negative), but that paracentesis was far superior as a diagnostic aid in lacerations of liver, spleen, or pancreas.

Keith et al. (1950) stated that it is often a useful investigation in acute pancreatitis. They obtained fluid in 12 out of 15 consecutive cases, and in all of these the amylase levels were higher than those found in the serum. Moreover, the peritoneal fluid amylase remained high for two to three days after the serum levels had returned to normal. However, Moretz and Erickson (1954) warn that peritoneal fluid amylase is also raised in perforated peptic ulcer and intestinal strangulation, and care must be taken in interpreting this finding.

The accuracy of the procedure was investigated experimentally by Giacobine and Siler (1960). Their observations on dogs showed that there was a linear relation between the amount of fluid in the peritoneal cavity and the probability of obtaining a sample by needle paracentesis. The low rate of positive results with small amounts of fluid was shown radiologically to be due to pooling in the pelvis, paravertebral gutters, and subphrenic spaces.

\section{Material and Methods}

The cases were an unselected series of 101 patients admitted as acute abdominal emergencies to a general hospital. There were 50 males and 51 females in the series, with an age range from 6 to 89 years.

\section{Technique}

The standard procedure throughout this investigation was the four-quadrant tap advocated by Byrne (1956). Though most authors advocate the use of a spinal puncture needle,

\footnotetext{
- Surgical Registrar, Central Middlesex Hospital, London. Present address: Middlesex Hospital, London W.1. t Consultant Surgeon, Central Middlesex Hospital, London.
}

entirely satisfactory results were obtained with a 2-ml. or 5-ml. disposable syringe and No. 12 needle. Local anaesthetic was not routinely employed and asepsis was confined to socially clean hands and a spirit skin preparation.

The needle was inserted at the sites shown in the Diagram. Light intermittent suction was applied as the needle tip was moved about within the peritoneal cavity, seeking pockets of free fluid. This is necessary to obtain a sample when only a small quantity of fluid is present as a thin film beneath the parietal peritoneum.

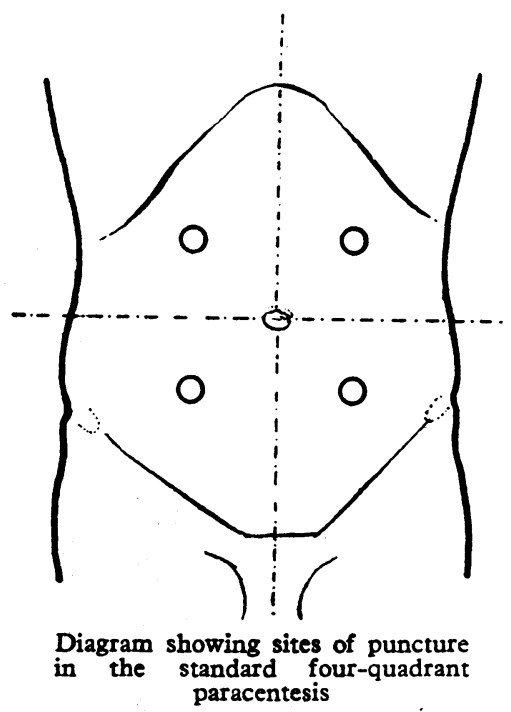

Occasionally a very small quantity (less than $0.5 \mathrm{ml}$.) of clear peritoneal fluid is obtained in the absence of intra-abdominal morbidity. When a larger quantity than this is obtained there is nearly always an underlying cause. For this reason we have regarded a paracentesis as "positive" if clear fluid in excess of $0.5 \mathrm{ml}$. has been obtained. If the fluid is obviously pathological-for example, pus-then the paracentesis is "positive" regardless of its volume.

The necessity of carrying out a full four-quadrant technique before pronouncing a paracentesis to be negative was demonstrated on several occasions in this series. In one case pus was obtained only from the right upper quadrant though the lesion was a perforated diverticulum of the sigmoid colon in the left lower quadrant. This also shows that the method gives no indication of the probable site of the primary lesion.

\section{Safety of the Technique}

The objection to the technique most often raised has been on the grounds of safety. This point has been investigated experimentally in dogs by Moretz and Erickson (1954). When isolated segments of intestine were deliberately punctured and subsequently inflated no leakage occurred until a pressure of $260 \mathrm{~mm}$. $\mathrm{Hg}$ was reached. Even in complete intestinal obstruction the intraluminal pressure seldom rises above $15 \mathrm{~mm}$. $\mathrm{Hg}$ in the small bowel and $20 \mathrm{~mm}$. $\mathrm{Hg}$ in the large bowel 
(Wangensteen, 1942). Leakage from accidental puncture is therefore a very small hazard. Nevertheless, in the presence of distended bowel the risk must be increased, and we believe that paracentesis should not be done in these circumstances.

No complication was recorded in any of the 101 cases reported, though visceral puncture did occur. The gall-bladder, the stomach, and the colon were each penetrated at some time. In those cases undergoing subsequent laparotomy no evidence of haemorrhage or peritoneal soiling was found. This confirms the findings of all previous authors. Indeed, the only complication recorded in the literature is of a haematoma of the rectus sheath after a paramedian tap (Drapanas and McDonald, 1961).

\section{Interpretation of a Positive Paracentesis}

Frank Blood.-The withdrawal of pure blood that fails to clot on standing can only mean that a significant intraperitoneal haemorrhage has occurred. Accidental puncture of a blood vessel does occur but can readily be distinguished by the fact that blood from this source clots within a few minutes.

Serosanguineous Fluid.-This type of exudate has been reported in a number of situations, including strangulated hernia (Hill et al., 1942) and acute pancreatitis (Keith et al., 1950). In the present series it was found in only two cases. One was a traumatic rupture of the kidney with a retroperitoneal haematoma, in which the paracentesis did not contribute towards the diagnosis. The other was a case of acute cholecystitis, described in detail below (Case 1).

Purulent Fluid.-This may vary from the offensive frank pus obtained from a perforated appendix or diverticulum of the colon to the thin turbid fluid associated with localized inflammatory disease. The latter contains numerous polymorphs on smearing the centrifuged deposit (see Case 1 below).

Bile-stained Fluid.-This type of fluid is characteristic of a perforated ulcer, and in the present series was the most common finding on paracentesis. Usually the fluid is turbid and contains food particles. A most important observation in our series was that the fluid was never acid when tested with ordinary litmus paper. Even when the sample was obtained very soon after the onset of acute symptoms the fluid was either neutral or alkaline. On one occasion the gall bladder was accidentally punctured in the right hypochondrium, yielding pure bile. This was correctly interpreted and no harm resulted.

Faecal.-When the intestine is accidentally punctured it is usually easy to recognize this by naked-eye inspection of the material aspirated. In cases of doubt, microscopy reveals a mixture of intestinal bacteria and debris with no evidence of polymorphs. No complications have resulted from this accident and there is no reason why the paracentesis should not be attempted with a fresh needle and syringe at a different site in the same quadrant.

\section{Misinterpretation of a Positive Paracentesis}

On two occasions a positive paracentesis was misinterpreted, and since each case illustrates a useful point they are described in detail.

Case 1.-An 87-year-old woman was admitted to hospital as an emergency with a history of severe upper abdominal pain of sudden onset. Examination revealed tenderness and guarding in the upper abdomen. A plain $x$-ray film showed no gas under the diaphragm. The serum amylase was less than 500 Somogyi units. On abdominal paracentesis a small quantity of serosanguineous fluid was obtained from the right hypochondrium. Microscopy of the centrifuged deposit showed numerous polymorphs, indicating the presence of an inflammatory lesion. Despite the nature of this fluid a clinical diagnosis of perforated peptic ulcer was made. At laparotomy the gall bladder was found to be acutely inflamed, and immediately surrounding it was a collection of free fluid identical to the aspirated sample. The stomach and duodenum were entirely normal.

Comment.-This is one of the two cases in which a positive paracentesis was obtained in the presence of local inflammatory disease. The point demonstrated is that where pathological fluid is obtained the diagnosis must be consistent with its type. Here the fluid was quite uncharacteristic of a perforated peptic ulcer, and this diagnosis should not have been made.

Case 2.-A man aged 62 was admitted to hospital with a history of upper abdominal pain for the preceding three days which had become much more severe that day. On admission there was clinical and radiographic evidence of bronchopneumonia. He was a known chronic bronchitic and was under treatment for recurrent episodes of congestive cardiac failure. However, there was also evidence of a perforated viscus, with marked tenderness over the whole abdomen and guarding in the epigastrium. Bowel sounds were absent. This diagnosis seemed confirmed when, on abdominal paracentesis, bile-stained fluid with food particles was obtained from the left iliac fossa. The $p \mathrm{H}$ of the fluid was not tested. All attempts to pass a Ryle tube failed. Since his condition was rapidly deteriorating it was decided to proceed with laparotomy. At operation no free fluid was found in the peritoneal cavity. The stomach was full of fluid and extended right down to the left iliac fossa. On its anterior surface was a puncture mark indicating that the attempted paracentesis had in fact withdrawn gastric contents. The patient died shortly after operation. At necropsy no cause was found for his abdominal symptoms. The only positive finding was an organizing bronchopneumonia, and it must be presumed that this was responsible for his entire clinical picture.

Comment.-This case has revealed a possible source of error that has not hitherto been reported. Two omissions probably account for the misinterpretation of the paracentesis. Firstly, if the $p \mathrm{H}$ of the fluid had been tested it would very probably have been acid. As stated above, fluid from a perforated peptic ulcer appears invariably to be either neutral or alkaline. Secondly, it proved impossible to pass a Ryle tube preoperatively. Had this been achieved, the large aspirate would have been noted and a repeat paracentesis would have proved negative. It therefore seems desirable always to test the $\mathrm{pH}$ of the fluid obtained from cases of suspected perforation. It would also seem more reliable to perform the paracentesis after gastric aspiration. However, this must be a very uncommon source of error.

\section{Interpretation of a Negative Paracentesis}

It cannot be too strongly emphasized that a negative paracentesis has no positive significance. If operation is indicated on clinical grounds, then, whatever the diagnosis may be, the fact that no fluid has been obtained from the peritoneal cavity must be completely disregarded.

If there is no clear indication for immediate surgery on clinical grounds, a negative paracentesis may help to justify continuation of conservative treatment, but it should be given no significance in arriving at a diagnosis.

\section{Results}

Of the 101 cases in which diagnostic paracentesis was performed, an acute abdominal condition was demonstrated at laparotomy or necropsy in 64 . Of four further cases presenting with severe abdominal pain, two were subsequently shown to have malignant disease, one had a coronary thrombosis, and one had tuberculosis of the urinary tract. Ascitic fluid was obtained from the former two cases; in the latter twe the paracentesis was negative.

There was therefore a total of 68 cases in which a firm diagnosis was made. In this group a positive paracentesis was 
obtained in 33 and a negative result in 35 . In Table I these results are analysed according to final diagnosis.

From these data a clear pattern emerges. Diagnostic paracentesis was found to yield accurate positive information in 22 . out of 28 cases of visceral perforation. No attempt was

TaBle I.-Abdominal Emergencies With Known Diagnosis

\begin{tabular}{l|l|c|c}
\hline & \multicolumn{3}{|c}{ Paracenteses } \\
\cline { 2 - 3 } & No. & Positive & Negative \\
\hline
\end{tabular}

\begin{tabular}{|c|c|c|c|c|}
\hline \multicolumn{2}{|r|}{ Group $I$. } & \multicolumn{2}{|c|}{ Visceral Perforations } & \multirow[b]{2}{*}{$\begin{array}{l}5 \\
0 \\
1\end{array}$} \\
\hline $\begin{array}{l}\text { Stomach and duodenum } \\
\text { Small bowel } \\
\text { Colon and appendix }\end{array}$ & $\ddot{*}$ & $\begin{array}{r}19 \\
2 \\
7\end{array}$ & $\begin{array}{r}14 \\
2 \\
6\end{array}$ & \\
\hline Total .. & . & 28 & $22(79 \%)$ & 6 \\
\hline
\end{tabular}
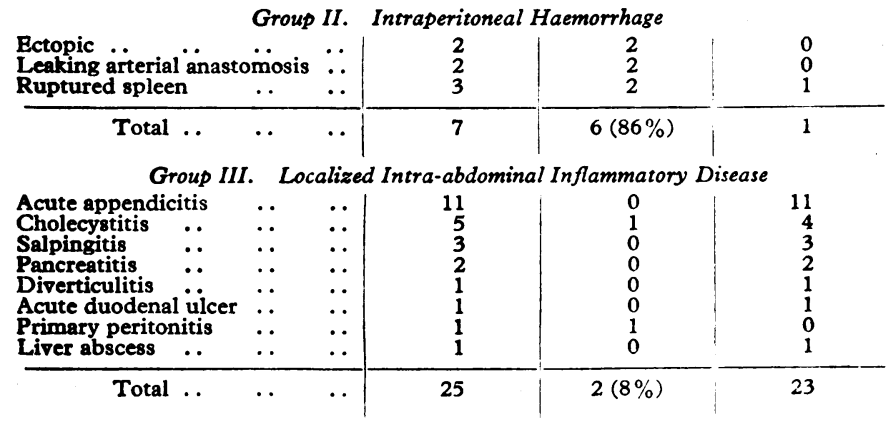

\begin{tabular}{ccc|c|c|c}
\multicolumn{7}{c}{ Group IV. Miscellaneous } \\
Intestinal obstruction &.. & $\ldots$ & 4 & 1 & \\
Malignant disease & $\ldots$ & $\ldots$ & 2 & 3 \\
Urinary tract tuberculosis & $\ldots$ & 1 & 2 & 0 \\
Coronary thrombosis &.. & $\ldots$ & 1 & 0 & 1 \\
\hline Total . . &.. & $\ldots$ & 8 & 3 & 1 \\
\hline Grand total &.. & $\ldots$ & 68 & 33 & 5 \\
\hline
\end{tabular}

made to compare the relative accuracy of this technique with that of diagnostic radiography, but it is unlikely that the latter method would have proved significantly better.

In cases of intraperitoneal haemorrhage blood was obtained in six out of seven paracenteses, a comparable degree of accuracy with the large series of such cases reported by Williams and Zollinger (1959).

In direct contrast to these findings are the results for localized inflammatory disease. Here it is quite clear that diagnostic paracentesis is most unlikely to give useful information. In only two cases out of 25 was a positive paracentesis obtained. One was the case of acute cholecystitis described above (Case 1). The other was a case of peritonitis, for which no cause was found at laparotomy and which was therefore classified as a primary peritonitis.

Only two cases of acute pancreatitis were encountered in this series, and no fluid was obtained from either of them.

Of the four patients with intestinal obstruction only one yielded a positive result. As stated above, the technique is not recommended for florid cases of obstruction, and in this series it was used only when the diagnosis was not clear. It may have a place in the diagnosis of intestinal strangulation (Hill et al., 1942), but the one such case in this series produced a negative result. At laparotomy there was a small amount of faeculent blood-stained fluid in the peritoneal cavity, but paracentesis had failed to detect it.

A recurrent problem in which the test may prove an outstanding aid to diagnosis is that of the postoperative patient who develops intra-abdominal complications. A recent anaesthetic, followed by the customary analgesia, makes the interpretation of physical signs notoriously difficult in these cases. Brief summaries of two such cases are given.

Case 3.-A 57-year-old man presented with an 18-month history of severe intermittent claudication of his left buttock and thigh after walking 25 yards (23 metres). An aortogram revealed athero- sclerotic occlusion at the aortic bifurcation. He underwent an aorto-iliac thrombendarterectomy on 21 February 1966. Two hours after the end of the operation he had a cardiac arrest in the ward. He was resuscitated from this successfully, but 15 minutes later his blood pressure again fell rapidly. At the time it was not clear whether this was due to myocardial damage or to bleeding from the aortic suture line. A diagnostic paracentesis was performed and non-clotting blood obtained from the right lower quadrant. On this evidence he was immediately returned to the theatre and the wound reopened. The diagnosis of leaking aortic suture line was confirmed (the peritoneal cavity containing some 2 litres of blood). Unfortunately the heart arrested again on the table before the bleeding could be properly controlled, and though it was restarted by open cardiac massage the patient did not survive.

Case 4.-A 52-year-old woman was admitted to hospital with a diagnosis of coronary thrombosis, and while on treatment suddenly developed the signs of an embolus at the aortic bifurcation. On 1 June 1966 an emergency aorto-iliac and bilateral femoral thrombectomy was performed. Her immediate postoperative condition was satisfactory, with a rapid return of the peripheral pulses in both legs. However, 12 hours later she began to deteriorate rapidly with signs of peripheral circulatory failure. It was thought likely that leakage had occurred from her arterial suture line, so a diagnostic paracentesis was performed. This produced heavily bloodstained fluid with a faecal odour from the right lower quadrant. She was taken back to the theatre and re-explored. At operation there was approximately 1 litre of blood in the peritoneal cavity, presumed to be due to a leak from the aortic suture line, though this was dry when exposed. However, the most significant finding was infarction of the colon, which accounted for the faeculent odour of the aspirate and which was undoubtedly the cause of the rapid deterioration in her condition. The inferior mesenteric artery was pulseless. An emergency colectomy was performed but the patient died a few hours later.

Comment.-Though unfortunately both these patients died, they do nevertheless illustrate that valuable information can be obtained from a diagnostic paracentesis in the early postoperative period when the critical decision has to be made whether or not to reoperate. Almost certainly in both these cases the correct decision would have been made eventually, but where speed of decision was essential no other single clinical investigation could have supplied the information so swiftly or so accurately.

\section{Undiagnosed Abdominal Pain}

There remains to be considered the group of 33 patients who were admitted with acute abdominal pain for which no cause was firmly established. They are analysed in Table II, and, broadly speaking, fall into two categories: (A) those with suspected acute intra-abdominal inflammatory disease; and (B) those with suspected intra-abdominal bleeding or a perforated viscus following blunt abdominal trauma.

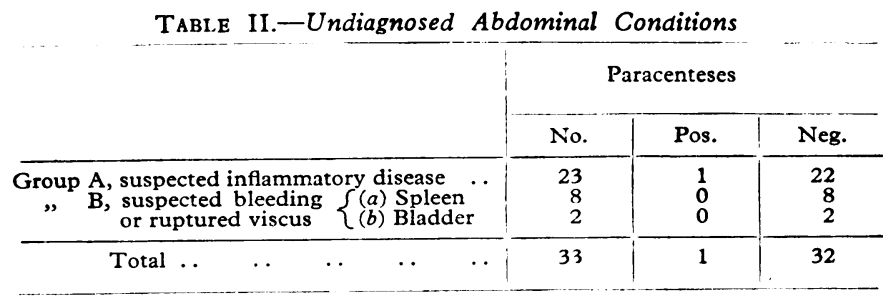

Of the 23 cases in group $\mathrm{A}$ the one positive paracentesis was obtained in a woman of 83 admitted with a clinical diagnosis of acute cholecystitis. Turbid fluid containing polymorphs was obtained from the right hypochondrium, which supported this diagnosis. Though the patient recovered on conservative treatment she was not regarded as a candidate for subsequent surgery, and the diagnosis was not confirmed radiologically.

Negative paracenteses were obtained in all 10 cases in group B. Only onc of these underwent operation. This was for a 
suspected traumatic rupture of the bladder, but laparotomy revealed only a retroperitoneal haematoma. The remaining nine cases all recovered on conservative treatment.

\section{Discussion}

This investigation has served to clarify the place of paracentesis in a variety of acute abdominal conditions.

In the first place the findings confirm the opinion of previous authors that the test is both simple and completely safe. We have also confirmed that a full four-quadrant technique is essential before a paracentesis can be regarded as negative.

Though the number of cases is small, the most accurate results have been obtained in suspected intraperitoneal haemorrhage. Since there is experimental evidence (Giacobine and Siler, 1960) that a volume of $500 \mathrm{ml}$. of free fluid in the peritoneal cavity will give a $78 \%$ positive paracentesis rate, the method is likely to be more sensitive and accurate than any other single diagnostic aid. We strongly recommend its use in all cases of blunt abdominal trauma, and particularly when the clinical picture is confused by the presence of other injuries.

In visceral perforations again a high yield of positive results was obtained, but in evaluating the method in this situation there are other factors to consider. Firstly, the clinical picture is usually much clearer than in intra-abdominal haemorrhage, so that in many cases ancillary aids to diagnosis are not required. Secondly, another simple diagnostic method-the plain erect and supine abdominal $x$-ray films-is available and also gives a high yield of positive results.

In cases where the clinician is in no doubt about the diagnosis, neither method is necessary and laparotomy need not be delayed. There are, however, many situations in which varying degrees of doubt exist regarding whether or not perforation has taken place. In these cases has paracentesis any advantage over $x$-ray examination as a method of diagnosis ?

In favour of paracentesis we can say: $(a)$ it is a test that can be performed immediately by the clinician at the bedside ; (b) it disturbs the patient far less than the manipulations required to obtain a satisfactory view of the erect abdomen; (c) it is an extremely useful test for hospitals lacking a 24-hour radiological service; and $(d)$ it gives a yield of positive information which is at least comparable to that of $x$-ray techniques.

By contrast, in localized inflammatory disease the yield of positive information is so low that abdominal paracentesis cannot be recommended as a useful diagnostic aid. The only exception to this may be in helping to differentiate between a localized inflammation and a perforated viscus, where the history and examination are equivocal.
Finally, we must repeat the warning, as have many previous authors, that a negative paracentesis has no significance. Serious underlying conditions may be present despite a negative tap. Our figures demonstrate that this is especially likely in localized inflammatory disease, but it is true also in intraperitoneal haemorrhage and visceral perforations.

In conclusion, therefore, we believe that diagnostic paracentesis is a useful procedure in the evaluation of patients with suspected intraperitoneal haemorrhage and visceral perforation, but has little place in the diagnosis of localized intra-abdominal inflammatory disease.

\section{Summary}

The value of diagnostic paracentesis has been studied in 101 patients admitted as cases of acute abdominal emergency.

The technique has been shown to be both simple and safe. Though visceral puncture may occasionally occur, no complications have resulted.

Analysis of the results shows that a high yield (approximately $80 \%$ ) of positive paracenteses can be expected in cases of intraperitoneal haemorrhage and perforated viscus. Conversely, a very low yield (less than $10 \%$ ) was obtained in localized inflammatory disease.

The test is strongly recommended as routine in all cases of blunt abdominal trauma. It is also extremely useful in the early diagnosis of complications after abdominal surgery.

In visceral perforation the method offers a useful alternative to radiological diagnosis, and in certain respects is to be preferred.

Our thanks are due to Mr. J. W. P. Gummer for permitting us to include his patients in this study.

\section{REFERENCES}

Byrne, R. V. (1956). West. F. Surg., 64, 369.

Drapanas, T., and McDonald, J. (1961). Surgery, 50, 742.

Economy, D., Koucky, C., and Novack, R. L. (1960). Amer. F. Surg., $99,647$.

Giacobine, J. W., and Siler, V. E. (1960). Surg. Gynec. Obstet., 110, 676

Hill, F. C., O'Loughlin, B. J., and Stoner, M. (1942). Ibid., 74, 121.

Keith, L. M., Zollinger, R. M., and McCleery, R. S. (1950). Arch. Surg., 61, 930 .

Moretz, W. H., and Erickson, W. G. (1954). Amer. Surgn, 20, 363.

Morris, P. J. (1966). Brit. f. Surg., 53, 707.

Neuhof, H., and Cohen, I. (1926). Ann. Surg., 83, 454.

Thompson, C. T., and Brown, D. R. (1954). Surgery, 35, 916.

Wangensteen, O. H. (1942). Intestinal Obstructions, 2nd ed. Springfield, Illinois.

Williams, R. D., and Zollinger, R. M. (1959). Amer. F. Surg., 97, 575. 